MATHEMATICS OF COMPUTATION

Volume 70, Number 233, Pages 367-387

S 0025-5718(00)01189-3

Article electronically published on June 12, 2000

\title{
TAMAGAWA NUMBERS OF DIAGONAL CUBIC SURFACES, NUMERICAL EVIDENCE
}

\author{
EMMANUEL PEYRE AND YURI TSCHINKEL
}

\begin{abstract}
A refined version of Manin's conjecture about the asymptotics of points of bounded height on Fano varieties has been developed by Batyrev and the authors. We test numerically this refined conjecture for some diagonal cubic surfaces.
\end{abstract}

\section{INTRODUCTION}

The aim of this paper is to test numerically a refined version of a conjecture of Manin concerning the asymptotic for the number of rational points of bounded height on Fano varieties (see [BM] or [FMT] for Manin's conjecture and [Pe1] or BT3 for its refined versions).

Let $V$ be a smooth Fano variety over a number field $F$ and $\omega_{V}^{-1}$ its anticanonical line bundle. Let $\operatorname{Pic}(V)$ be the Picard group and $\mathrm{NS}(V)$ the Néron-Severi group of $V$. We denote by $\operatorname{Val}(F)$ the set of all places of $F$ and by $F_{v}$ the $v$-adic completion of $F$. Let $\left(\|\cdot\|_{v}\right)_{v \in \operatorname{Val}(F)}$ be an adelic metric on $\omega_{V}^{-1}$. By definition, this is a family of $v$-adically continuous metrics on $\omega_{V}^{-1} \otimes F_{v}$ which for almost all valuations $v$ are given by a smooth model of $V$ (see $[\mathrm{Pe} 2])$. These data define a height $\mathbf{H}$ on the set of rational points $V(F)$ given by

$$
\forall x \in V(F), \quad \forall y \in \omega_{V}^{-1}(x), \quad \mathbf{H}(x)=\prod_{v \in \operatorname{Val}(F)}\|y\|_{v}^{-1} .
$$

For every open subset $U \subset V$ and every real number $H$ we have

$$
n_{U, \mathbf{H}}(H)=\#\{x \in U(F) \mid \mathbf{H}(x) \leqslant H\}<\infty .
$$

The problem is to understand the asymptotic behavior of $n_{U \cdot \mathbf{H}}(H)$ as $H$ goes to infinity. It is expected that at least for Del Pezzo surfaces the following asymptotic formula holds:

$$
n_{U, \mathbf{H}}(H)=\boldsymbol{\theta}_{\mathbf{H}}(V) H(\log H)^{t-1}(1+o(1))
$$

as $H \rightarrow \infty$, over appropriate finite extensions $E / F$ of the groundfield. Here the open set $U$ is the complement to exceptional curves, $\boldsymbol{\theta}_{\mathbf{H}}(V)>0$, and $t$ is the rank of

Received by the editor June 22, 1998 and in revised form, January 4, 1999.

2000 Mathematics Subject Classification. Primary 11D25, 14G40; Secondary 14G05, 14J25.

(C)2000 American Mathematical Society 
the Picard group of $V$ over $E$. We have counterexamples to this conjecture in every dimension $\geqslant 3$ BT2] (see BT3 for a discussion of higher dimensional varieties).

In this paper we focus on the constant $\boldsymbol{\theta}_{\mathbf{H}}(V)$. On the one hand, there is a theoretical description

$$
\boldsymbol{\theta}_{\mathbf{H}}(V)=\alpha(V) \beta(V) \tau_{\mathbf{H}}(V),
$$

where $\tau_{\mathbf{H}}(V)$ is a Tamagawa number associated to the metrized anticanonical line bundle [ $\mathrm{Pe} 1, \alpha(V)$ is a rational number defined in terms of the cone of effective divisors [Pe1, and the integer $\beta(V)$ is a cohomological invariant which first appeared in asymptotic formulas in BT1].

On the other hand, let us consider a diagonal cubic surface $V \subset \mathbf{P}_{\mathbf{Q}}^{3}$ given by

$$
a x^{3}+b y^{3}+c z^{3}+d t^{3}=0,
$$

with $a, b, c, d \in \mathbf{Z}$ and $a b c d \neq 0$. Our counting problem can be formulated as follows. Find all quadruples of integers $(x, y, z, t)$ with

$$
\text { g.c.d. }(x, y, z, t)=1 \quad \text { and } \max \{|x|,|y|,|z|,|t|\} \leqslant H
$$

which satisfy the equation above. Quadruples differing by a sign are counted once. A proof of an asymptotic of the type (1) for smooth cubic surfaces seems to be out of the reach of available methods, but one can numerically search for solutions of bounded height. The cubics with coefficients $(1,1,1,2)$ and $(1,1,1,3)$ and height $H \leqslant 2000$ were treated by Heath-Brown in $[\mathrm{H}-\mathrm{B}$. In both cases weak approximation fails. Swinnerton-Dyer made substantial progress toward an interpretation of the constant $\tau_{\mathbf{H}}(V)$ S-D. In particular, he suggested that the adelic integral defining $\tau_{\mathbf{H}}(V)$ should be taken over the closure of rational points $\overline{V(F)} \subset V\left(A_{F}\right)$, rather than the whole adelic space.

Our goal is to compute the theoretical constant $\boldsymbol{\theta}_{\mathbf{H}}(V)$ explicitly for certain diagonal cubic surfaces with and without obstruction to weak approximation, and to compare the result with numerical data (with height $H \leqslant 100000$ ). We observe a very good accordance.

In Section 2 we define the Tamagawa number. This definition is slightly different from the one in [Pe1], but the numbers coincide conjecturally. In Sections 3, 4 and 5 we explain how to compute it. There is a subtlety at the places of bad reduction, notable at 3, overlooked previously. In Section 6 we compute the Brauer-Manin obstruction to weak approximation. And in Section 7 we present the numerical results. These computations were made using a program of Bernstein which is described in $[\mathrm{Be}$.

\section{Conjectural constant}

Notations 2.1. If $\mathcal{V}$ is a scheme over a ring $A$ and $B$ an $A$-algebra, we denote by $\mathcal{V}_{B}$ the product $\mathcal{V} \times \times_{\operatorname{Spec} A} \operatorname{Spec} B$ and by $\mathcal{V}(B)$ the set of $B$-points, that is $\operatorname{Hom}_{\operatorname{Spec} A}(\operatorname{Spec} B, \mathcal{V})$. For any field $E$, we denote by $\bar{E}$ a fixed algebraic closure and by $\bar{V}$ the variety $V_{\bar{E}}$.

If $F$ is a number field, we identify the set of finite places with the set of prime ideals in $\mathcal{O}_{F}$. We denote by $d_{F}$ the absolute value of its discriminant. If $\mathfrak{p}$ is a finite 
place of $F$, then $\mathcal{O}_{\mathfrak{p}}$ is the ring of integers in $F_{\mathfrak{p}}$ and $\mathbf{F}_{\mathfrak{p}}$ its residue field.

We will always assume that $V$ is a smooth projective geometrically integral variety over a number field $F$ satisfying the following conditions:

(i) The group $H^{i}\left(V, \mathcal{O}_{V}\right)$ is trivial for $i=1$ or 2 ;

(ii) $\operatorname{Pic}(\bar{V})$ has no torsion;

(iii) $\omega_{V}^{-1}$ belongs to the interior of the cone of classes of effective divisors $\Lambda_{\text {eff }}(V)$.

Since $V$ is projective, the adelic space $V\left(A_{F}\right)$ of $V$ coincides with the product $\prod_{v \in \operatorname{Val}(F)} V\left(F_{v}\right)$. One says that weak approximation holds for $V$ if the diagonal map from $V(F)$ to $V\left(A_{F}\right)$ has a dense image. Our definition of the conjectural asymptotic constant $\boldsymbol{\theta}_{\mathbf{H}}(V)$ uses the notion of the Brauer-Manin obstruction to weak approximation, which we now recall.

Notations 2.2. Let $\operatorname{Br}(V)$ be the étale cohomology group $H_{\text {ét }}^{2}\left(V, \mathbf{G}_{m}\right)$. If $A$ belongs to $\operatorname{Br}(V)$ and $E$ is a field over $F$, then for any $P$ in $V(E)$ we denote by $A(P)$ the evaluation of $A$ at $P$. For any class $A$, there exists a finite set of places $S$ of $F$ such that

$$
\forall v \notin S, \quad \forall P_{v} \in V\left(F_{v}\right), \quad A\left(P_{v}\right)=0
$$

(see, for example, CT2 Lemma 1]). For any $v$ in $\operatorname{Val}(F)$, let $\operatorname{inv}_{v}: \operatorname{Br}\left(F_{v}\right) \rightarrow \mathbf{Q} / \mathbf{Z}$ be the invariant given by local class field theory normalized so that the sequence

$$
0 \rightarrow \operatorname{Br}(F) \rightarrow \bigoplus_{v \in \operatorname{Val}(F)} \operatorname{Br}\left(F_{v}\right) \stackrel{\Sigma \operatorname{inv}_{v}}{\longrightarrow} \mathbf{Q} / \mathbf{Z} \rightarrow 0
$$

is exact. Let $\rho_{A}$ be the composite map

$$
V\left(A_{F}\right) \rightarrow \bigoplus_{v \in \operatorname{Val}(F)} \operatorname{Br}\left(F_{v}\right) \stackrel{\Sigma \operatorname{inv}_{v}}{\longrightarrow} \mathbf{Q} / \mathbf{Z}
$$

Then one defines

$$
V\left(A_{F}\right)^{\mathrm{Br}}=\bigcap_{A \in \operatorname{Br} V} \operatorname{ker}\left(\rho_{A}\right) \subset V\left(A_{F}\right) .
$$

The above exact sequence gives an inclusion $V(F) \subset V\left(A_{F}\right)^{\mathrm{Br}}$. The Brauer-Manin obstruction to weak approximation, introduced by Manin in Ma1 and by ColliotThélène and Sansuc in [CTS] is defined as the condition

$$
V\left(A_{F}\right)^{\mathrm{Br}} \varsubsetneqq V\left(A_{F}\right) .
$$

Remark 2.1. It is conjectured that the closure of the set of rational points $\overline{V(F)} \subset$ $V\left(A_{F}\right)$ in fact coincides with $V\left(A_{F}\right)^{\mathrm{Br}}$, at least for Del Pezzo surfaces. This has been proved, for example, by Salberger and Skorobogatov for a smooth complete intersection of two quadrics in $\mathbf{P}^{4}$ if $V(F)$ is not empty (see [SaSk]). It would be very interesting to see an example of a cubic surface $V$ with $t=\operatorname{rk} \operatorname{Pic}(V)=1$ where weak approximation holds, or where one could actually prove that $\overline{V(F)}=V\left(A_{F}\right)^{\mathrm{Br}}$, assuming that $V(F)$ is Zariski dense, which by a result of B. Segre (see [Ma2, $\S 29$, $\S 30])$ is equivalent to $V(F) \neq \emptyset$.

Notations 2.3. Let $\left(\|\cdot\|_{v}\right)_{v \in \operatorname{Val}(F)}$ be an adelic metric on $\omega_{V}^{-1}$ and $\mathbf{H}$ the associated height function on $V(F)$. The adelic metrization of the anticanonical line bundle 
yields for any place $v$ of $F$ a measure $\boldsymbol{\omega}_{\mathbf{H}, v}$ on the locally compact space $V\left(F_{v}\right)$, given by the local formula

$$
\boldsymbol{\omega}_{\mathbf{H}, v}=\left\|\frac{\partial}{\partial x_{1, v}} \wedge \cdots \wedge \frac{\partial}{\partial x_{n, v}}\right\|_{v} \mathrm{~d} x_{1, v} \cdots \mathrm{d} x_{n, v},
$$

where $x_{1, v}, \ldots, x_{n, v}$ are local $v$-adic analytic coordinates, $\frac{\partial}{\partial x_{1, v}} \wedge \cdots \wedge \frac{\partial}{\partial x_{n, v}}$ is seen as a section of $\omega_{V}^{-1}$, and the Haar measures $\mathrm{d} x_{j, v}($ for $j=1, \ldots, n)$ are normalized by

- if $v$ is a finite place, then $\int_{\mathcal{O}_{v}} \mathrm{~d} x_{j, v}=1$;

- if $v$ is real, then $\mathrm{d} x_{j, v}$ is the standard Lebesgue measure;

- if $v$ is complex, then $\mathrm{d} x_{j, v}=\mathrm{d} z \mathrm{~d} \bar{z}$.

We choose a finite set $S$ of bad places containing the archimedean ones and a smooth projective model $\mathcal{V}$ of $V$ over the ring of $S$-integers $\mathcal{O}_{S}$. For any $\mathfrak{p}$ in $\operatorname{Val}(F)-S$, the local term of the $L$-function corresponding to the Picard group is defined by

$$
L_{\mathfrak{p}}(s, \operatorname{Pic}(\bar{V}))=\frac{1}{\operatorname{Det}\left(1-\left(\# \mathbf{F}_{\mathfrak{p}}\right)^{-s} \operatorname{Fr} \mid \operatorname{Pic}\left(\mathcal{V}_{\overline{\mathbf{F}}_{\mathfrak{p}}}\right) \otimes \mathbf{Q}\right)},
$$

where Fr is the Frobenius map. The corresponding global $L$-function is given by

$$
L_{S}(s, \operatorname{Pic}(\bar{V}))=\prod_{\mathfrak{p} \in \operatorname{Val}(F)-S} L_{\mathfrak{p}}(s, \operatorname{Pic}(\bar{V})),
$$

it converges for $\operatorname{Re}(s)>1$ and has a meromorphic continuation to $\mathbf{C}$ with a pole of order $t=\operatorname{rk} \operatorname{Pic}(V)$ at 1 . The local convergence factors are defined by

$$
\lambda_{v}=\left\{\begin{array}{l}
L_{v}(1, \operatorname{Pic}(\bar{V})) \text { if } v \in \operatorname{Val}(F)-S, \\
1 \text { otherwise }
\end{array}\right.
$$

The Weil conjectures, proved by Deligne, imply that the adelic measure

$$
\prod_{v \in \operatorname{Val}(F)} \lambda_{v}^{-1} \omega_{\mathbf{H}, v}
$$

converges on $V\left(A_{F}\right)$ (see [Pe1, Proposition 2.2.2]).

Definition 2.4. The Tamagawa measure corresponding to $\mathbf{H}$ is defined by

$$
\boldsymbol{\omega}_{\mathbf{H}}=\frac{1}{\sqrt{d_{F}^{\operatorname{dim} V}}} \lim _{s \rightarrow 1}(s-1)^{t} L_{S}(s, \operatorname{Pic}(\bar{V})) \prod_{v \in \operatorname{Val}(F)} \lambda_{v}^{-1} \boldsymbol{\omega}_{\mathbf{H}, v}
$$

The Tamagawa number is defined by

$$
\tau_{\mathbf{H}}(V)=\boldsymbol{\omega}_{\mathbf{H}}\left(V\left(A_{F}\right)^{\mathrm{Br}}\right) .
$$

The cohomological constant is given by

$$
\beta(V)=\# H^{1}(F, \operatorname{Pic}(\bar{V})) .
$$


Let $\operatorname{NS}(V)^{\vee}$ be the lattice dual to $\operatorname{NS}(V)$. It defines a natural Lebesgue measure dy on $\operatorname{NS}(V)^{\vee} \otimes \mathbf{R}$. Denote by $\Lambda_{\text {eff }}(V) \subset \mathrm{NS}(V) \otimes \mathbf{R}$ the cone of effective divisors and by $\Lambda_{\text {eff }}(V)^{\vee} \subset \mathrm{NS}(V)^{\vee} \otimes \mathbf{R}$ the dual cone.

Definition 2.5. We define

$$
\alpha(V)=\frac{1}{(t-1) !} \int_{\Lambda_{\text {eff }}(V)^{\vee}} e^{-\left\langle\omega_{V}^{-1}, \mathbf{y}\right\rangle} \mathbf{d y} .
$$

Remarks 2.2. (i) Of course, for nonsplit cubic surfaces with $\operatorname{rk} \operatorname{Pic}(V)=1$ the constant $\alpha(V)=1$. However, it is a challenge to compute this constant for a split cubic surface with $\operatorname{rk} \operatorname{Pic}(V)=7$.

(ii) As Salberger, we use $\boldsymbol{\omega}_{\mathbf{H}}\left(V\left(A_{F}\right)^{\mathrm{Br}}\right)$ instead of $\boldsymbol{\omega}_{\mathbf{H}}(\overline{V(k)})$ in the definition of $\tau_{\mathbf{H}}(V)$. By Remark 2.1 these numbers are conjecturally the same, but only the first one is computable for a general cubic. Also we use the convention of [Pe1, §2.2.5] for the definition of $\alpha(V)$.

Definition 2.6. We define the constant corresponding to $V$ and $\mathbf{H}$ as

$$
\boldsymbol{\theta}_{\mathbf{H}}(V)=\alpha(V) \beta(V) \tau_{\mathbf{H}}(V) .
$$

\section{Measures AND DENSity}

In this section we relate the local volumes of the variety with the density of solutions modulo $\mathfrak{p}^{n}$. Lemma 5.4.6 in [Pe1] relates the local volume for $\boldsymbol{\omega}_{\mathbf{H , \mathfrak { p }}}$ to the volume for Leray's measure. We now compare the latter to the density modulo $\mathfrak{p}^{n}$.

Notations 3.1. Let $F$ be a number field and $V$ a smooth complete intersection in $\mathbf{P}_{F}^{N}$ defined by $m$ homogeneous polynomials $f_{i}$ in the algebra $\mathcal{O}_{F}\left[X_{0}, \ldots, X_{N}\right]$. Let $\delta=N+1-\sum_{i=1}^{m} \operatorname{deg} f_{i}$. We assume that $\delta \geqslant 1$. We denote by $W \subset \mathbf{A}_{F}^{N+1}-\{0\}$ the cone above $V$ and by $f: \mathbf{A}_{\mathcal{O}_{F}}^{N+1} \rightarrow \mathbf{A}_{\mathcal{O}_{F}}^{m}$ the map induced by the $f_{i}$. Then the Leray form on $W$ is defined locally by

$$
\begin{aligned}
\omega_{L}=(-1)^{N m-\sum_{j=1}^{m} k_{j}}(\operatorname{Det} & \left.\left(\frac{\partial f_{i}}{\partial X_{k_{j}}}\right)_{1 \leqslant i, j \leqslant m}\right)^{-1} \\
& \times \mathrm{d} X_{0} \wedge \cdots \wedge \widehat{\mathrm{d} X_{k_{1}}} \wedge \cdots \wedge \widehat{\mathrm{d} \widehat{X_{k_{m}}}} \wedge \cdots \wedge \mathrm{d} X_{N}
\end{aligned}
$$

where $0 \leqslant k_{1}<\cdots<k_{m} \leqslant N$. For any $v$ in $\operatorname{Val}(F)$, this form yields a measure $\boldsymbol{\omega}_{L, v}$ on $W\left(F_{v}\right)$.

The following result is well known in the setting of the circle method (see for example [Lac, Proposition 1.14]) where it is generally proved using a Fourier inversion formula. It may also be deduced from a more general result of Salberger $\mathrm{Sa}$, Theorem 2.13]. We prove it here in a direct and elementary way.

Proposition 3.1. We fix a finite place $v=v_{\mathfrak{p}}$ of $F$. If all $f_{i}$ have the same degree, then

$$
\int_{\left\{x \in \mathcal{O}_{\mathfrak{p}}^{N+1} \mid f(x)=0\right\}} \underset{\boldsymbol{\omega}_{L, \mathfrak{p}}}{ }=\lim _{r \rightarrow+\infty} \frac{\#\left\{x \in\left(\mathcal{O}_{\mathfrak{p}} / \mathfrak{p}^{r}\right)^{N+1} \mid f(x)=0 \text { in }\left(\mathcal{O}_{\mathfrak{p}} / \mathfrak{p}^{r}\right)^{m}\right\}}{\left(\# \mathbf{F}_{\mathfrak{p}}\right)^{r \operatorname{dim} W}}
$$


This proposition follows from the next two lemmata. In fact, in the explicit computations, we shall only use Lemma 3.2 and the first assertion of Lemma 3.4

Lemma 3.2. For any $r>0$, we consider the set

$$
W^{*}\left(\mathcal{O}_{\mathfrak{p}} / \mathfrak{p}^{r}\right)=\left\{x \in\left(\mathcal{O}_{\mathfrak{p}} / \mathfrak{p}^{r}\right)^{N+1}-\left(\mathfrak{p} / \mathfrak{p}^{r}\right)^{N+1} \mid f(x)=0 \text { in }\left(\mathcal{O}_{\mathfrak{p}} / \mathfrak{p}^{r}\right)^{m}\right\}
$$

and put $N^{*}\left(\mathfrak{p}^{r}\right)=\# W^{*}\left(\mathcal{O} / \mathfrak{p}^{r}\right)$. Then there is an integer $r_{0}>0$ such that

$$
\int_{\left\{x \in \mathcal{O}_{\mathfrak{p}}^{N+1}-\mathfrak{p}^{N+1} \mid f(x)=0\right\}}^{\boldsymbol{\omega}_{L, \mathfrak{p}}}=\frac{N^{*}\left(\mathfrak{p}^{r}\right)}{\left(\# \mathbf{F}_{\mathfrak{p}}\right)^{r \operatorname{dim} W}}
$$

if $r \geqslant r_{0}$.

Remark 3.3. It will follow from the proof that it is in fact sufficient to take $r_{0}$ to be

$$
2 \inf \left\{r \in \mathbf{Z}_{>0} \mid \forall x \in \mathcal{O}_{\mathfrak{p}}^{N+1}-\mathfrak{p}^{N+1}, f(x) \equiv 0 \bmod \mathfrak{p}^{r} \Rightarrow\left(\mathfrak{p}^{r}\right)^{m} \subset \operatorname{Im}\left(\mathrm{d} f_{x}\right)\right\}+1 .
$$

Proof. For any $r>0$,

$$
\begin{aligned}
& \int_{\left\{x \in \mathcal{O}_{\mathfrak{p}}^{N+1}-\mathfrak{p}^{N+1} \mid f(x)=0\right\}}^{\boldsymbol{\omega}_{L, \mathfrak{p}}}=\sum_{x \in\left(\mathcal{O}_{\mathfrak{p}} / \mathfrak{p}^{r}\right)^{N+1}-\left(\mathfrak{p} / \mathfrak{p}^{r}\right)^{N+1}} \int_{\left\{y \in \mathcal{O}_{\mathfrak{p}}^{N+1} \mid f(y)=0 \text { and }[y]_{r}=x\right\}} \boldsymbol{\omega}_{L, \mathfrak{p}}(y) \\
&=\sum_{x \in W^{*}\left(\mathcal{O}_{\mathfrak{p}} / \mathfrak{p}^{r}\right)} \int_{\left\{y \in \mathcal{O}_{\mathfrak{p}}^{N+1} \mid f(y)=0 \text { and }[y]_{r}=x\right\}} \\
& \boldsymbol{\omega}_{L, \mathfrak{p}}(y)
\end{aligned}
$$

where for any $y$ in $\mathcal{O}_{\mathfrak{p}}^{N+1}$ we denote by $[y]_{r}$ its class modulo $\mathfrak{p}^{r}$. Since $V$ is smooth, the cone $W$ does not intersect the cone defined by the equations

$$
\operatorname{det}\left(\frac{\partial f_{i}}{\partial X_{k_{j}}}\right)_{1 \leqslant i, j \leqslant m}=0 \text { for } 0 \leqslant k_{1}<\cdots<k_{m} \leqslant N \text {. }
$$

Therefore, for $r$ big enough and for any $x$ in $\left(\mathcal{O}_{\mathfrak{p}} / \mathfrak{p}^{r}\right)^{N+1}-\left(\mathfrak{p} / \mathfrak{p}^{r}\right)^{N+1}$ such that $f(x)=0$ in $\left(\mathcal{O}_{\mathfrak{p}} / \mathfrak{p}^{r}\right)^{m}$ one has that

$$
\inf _{\left(k_{j}\right)_{j}} v_{\mathfrak{p}}\left(\operatorname{det}\left(\frac{\partial f_{i}}{\partial X_{k_{j}}}\right)_{1 \leqslant i, j \leqslant m}\right)
$$

is finite and constant on the class defined by $x$. Let $c$ be its value. We may assume that $r>c$ and choose a family $0 \leqslant k_{1}<\cdots<k_{m} \leqslant N$ which realizes this minimum. We may assume that $k_{j}=N-m+j$. Then if $y \in \mathcal{O}_{\mathfrak{p}}^{N+1}$ represents $x$ and if $z$ belongs to $\mathcal{O}_{\mathfrak{p}}^{N+1}$, one has

$$
f_{i}(y+z)=f_{i}(y)+\sum_{j=0}^{N} \frac{\partial f_{i}}{\partial X_{j}}(y) z_{j}+\sum_{j, k=0}^{N} P_{i, j, k}(y, z) z_{j} z_{k},
$$

where the $P_{i, j, k}$ are polynomials in $2 N+2$ variables with coefficients in $\mathcal{O}_{\mathfrak{p}}$. Let $L_{y}$ be the image of the linear map defined by $\left(\frac{\partial f_{i}}{\partial X_{j}}(y)\right)$ on $\left(\mathcal{O}_{\mathfrak{p}}\right)^{N+1}$, then one has the inclusions

$$
\left(\mathfrak{p}^{r}\right)^{m} \subset\left(\mathfrak{p}^{c}\right)^{m} \subset L_{y} \subset\left(\mathcal{O}_{\mathfrak{p}}\right)^{m}
$$


and $\#\left(\left(\mathcal{O}_{\mathfrak{p}}\right)^{m} / L_{y}\right)=\left(\# \mathbf{F}_{\mathfrak{p}}\right)^{c}$. In particular, for any $z$ in $\left(\mathfrak{p}^{r}\right)^{N+1}$ one has $L_{y+z}=$ $L_{y}$. We put $L=L_{y}$. By (3) we have that for any $z$ in $\left(\mathfrak{p}^{r}\right)^{N+1}$,

$$
f(y+z)-f(y) \in \mathfrak{p}^{r} L .
$$

Therefore, the image of $f(y)$ in $\mathcal{O}_{\mathfrak{p}}^{m} / \mathfrak{p}^{r} L$ depends only on $x$ and we denote it by $f^{*}(x)$. If $f^{*}(x) \neq 0$, then the set

$$
\left\{u \in \mathcal{O}_{\mathfrak{p}}^{N+1} \mid f(u)=0 \text { and }[u]_{r}=x\right\}
$$

is empty and the integral is trivial. On the other hand, the set

$$
\left\{u \in\left(\mathcal{O}_{\mathfrak{p}} / \mathfrak{p}^{r+c}\right)^{N+1} \mid f(u)=0 \text { in }\left(\mathcal{O}_{\mathfrak{p}} / \mathfrak{p}^{r+c}\right)^{m} \text { and }[u]_{r}=x\right\}
$$

is also empty. If $f^{*}(x)=0$, then it follows from Hensel's lemma that the coordinates $X_{0}, \ldots, X_{N-m}$ define an isomorphism from

$$
\left\{u \in \mathcal{O}_{\mathfrak{p}}^{N+1} \mid f(u)=0 \text { and }[u]_{r}=x\right\}
$$

to $\left(y_{0}, \ldots, y_{N-m}\right)+\left(\mathfrak{p}^{r}\right)^{N-m+1}$. Therefore, using (2) and the definition of $c$, we get that

$$
\begin{aligned}
\int_{\left\{y \in \mathcal{O}_{\mathfrak{p}}^{N+1} \mid f(y)=0 \text { and }[y]_{r}=x\right\}} & \int_{\left(y_{0}, \ldots, y_{N-m}\right)+\left(\mathfrak{p}^{r}\right)^{N-m+1}}\left(\# \mathbf{F}_{\mathfrak{p}}\right)^{c} \mathrm{~d} u_{0, \mathfrak{p}} \ldots \mathrm{d} u_{N-m, \mathfrak{p}} \\
& =\# \mathbf{F}_{\mathfrak{p}}^{c-r} \operatorname{dim} W
\end{aligned}
$$

Let $x / \mathfrak{p}^{r+c}$ be the set

$$
\left\{u \in\left(\mathcal{O}_{\mathfrak{p}} / \mathfrak{p}^{r+c}\right)^{N+1} \mid[u]_{r}=x\right\} .
$$

Then $f$ induces a map from $x / \mathfrak{p}^{r+c}$ to $\left(\mathcal{O}_{\mathfrak{p}} / \mathfrak{p}^{r+c}\right)^{m}$ given by

$$
f\left([y+z]_{r+c}\right)=[f(y)]_{r+c}+\sum_{j=0}^{N} \frac{\partial f}{\partial X_{j}}(y) z_{j}
$$

the image of which is $\mathfrak{p}^{r} L /\left(\mathfrak{p}^{r+c}\right)^{m}$. Therefore, we obtain

$$
\begin{aligned}
\#\left\{u \in x / \mathfrak{p}^{r+c} \mid f(u)=0 \text { in }\left(\mathcal{O}_{\mathfrak{p}} / \mathfrak{p}^{r+c}\right)^{m}\right\} & \\
& =\#\left(\mathfrak{p}^{r} L /\left(\mathfrak{p}^{r+c}\right)^{m}\right)^{-1} \times \#\left(\mathfrak{p}^{r} / \mathfrak{p}^{r+c}\right)^{N+1} \\
& =\# \mathbf{F}_{\mathfrak{p}}^{c+c \operatorname{dim} W}
\end{aligned}
$$

and

$$
\frac{\#\left\{u \in x / \mathfrak{p}^{r+c} \mid f(u)=0 \operatorname{in}\left(\mathcal{O}_{\mathfrak{p}} / \mathfrak{p}^{r+c}\right)^{m}\right\}}{\# \mathbf{F}_{\mathfrak{p}}^{(r+c) \operatorname{dim} W}}=\# \mathbf{F}_{\mathfrak{p}}^{c-r \operatorname{dim} W} .
$$

Finally, we get the result.

Lemma 3.4. With notation as in Proposition 3.1, one has

$$
\int_{\left\{x \in \mathcal{O}_{\mathfrak{p}}^{N+1}-\mathfrak{p}^{N+1} \mid f(x)=0\right\}} \underset{\boldsymbol{\omega}_{L, \mathfrak{p}}}{ }=\left(1-\frac{1}{\# \mathbf{F}_{\mathfrak{p}}^{\delta}}\right) \int_{\left\{x \in \mathcal{O}_{\mathfrak{p}}^{N+1} \mid f(x)=0\right\}} \boldsymbol{\omega}_{L, \mathfrak{p}}
$$


and

$$
\begin{aligned}
& \lim _{r \rightarrow+\infty} \frac{N^{*}\left(\mathfrak{p}^{r}\right)}{\left(\# \mathbf{F}_{\mathfrak{p}}\right)^{r \operatorname{dim} W}} \\
& \quad=\left(1-\frac{1}{\# \mathbf{F}_{\mathfrak{p}}^{\delta}}\right) \lim _{r \rightarrow+\infty} \frac{\#\left\{x \in\left(\mathcal{O}_{\mathfrak{p}} / \mathfrak{p}^{r}\right)^{N+1} \mid f(x)=0 \text { in }\left(\mathcal{O}_{\mathfrak{p}} / \mathfrak{p}^{r}\right)^{m}\right\}}{\left(\# \mathbf{F}_{\mathfrak{p}}\right)^{r \operatorname{dim} W} .}
\end{aligned}
$$

Proof. By definition, one has for any $\lambda$ in $F_{\mathfrak{p}}^{*}$ the relation

$$
\boldsymbol{\omega}_{L, \mathfrak{p}}(\lambda U)=|\lambda|_{\mathfrak{p}}^{\delta} \boldsymbol{\omega}_{L, \mathfrak{p}}(U)
$$

which implies the first assertion. For the second one, let $d$ be the common degree of the $f_{i}$. If $r \geqslant i d+1$, one has the relations

$$
\begin{aligned}
\#\{x & \left.\in\left(\mathfrak{p}^{i} / \mathfrak{p}^{r}\right)^{N+1}-\left(\mathfrak{p}^{i+1} / \mathfrak{p}^{r}\right)^{N+1} \mid f(x) \equiv 0 \bmod \mathfrak{p}^{r}\right\} \\
& =\#\left\{x \in\left(\mathcal{O}_{\mathfrak{p}} / \mathfrak{p}^{r-i}\right)^{N+1}-\left(\mathfrak{p} / \mathfrak{p}^{r-i}\right)^{N+1} \mid f(x) \equiv 0 \bmod \mathfrak{p}^{r-i d}\right\} \\
& =\# \mathbf{F}_{\mathfrak{p}}^{(N+1)(d-1) i} \#\left\{x \in\left(\mathcal{O}_{\mathfrak{p}} / \mathfrak{p}^{r-i d}\right)^{N+1}-\left(\mathfrak{p} / \mathfrak{p}^{r-i d}\right)^{N+1} \mid f(x) \equiv 0 \bmod \mathfrak{p}^{r-i d}\right\} .
\end{aligned}
$$

Thus we get

$$
\begin{aligned}
& \#\left\{x \in\left(\mathcal{O}_{\mathfrak{p}} / \mathfrak{p}^{r}\right)^{N+1} \mid f(x)=0 \text { in }\left(\mathcal{O}_{\mathfrak{p}} / \mathfrak{p}^{r}\right)^{m}\right\} \\
& =\sum_{0 \leqslant i \leqslant a} \# \mathbf{F}_{\mathfrak{p}}^{(N+1)(d-1) i} N^{*}\left(\mathfrak{p}^{r-i d}\right) \\
& \quad+\#\left\{x \in\left(\mathfrak{p}^{a+1} / \mathfrak{p}^{r}\right)^{N+1} \mid f(x) \equiv 0 \bmod \mathfrak{p}^{r}\right\},
\end{aligned}
$$

where $r-r_{0}=a d+b$ with $b<d$. We have $a>\left(r-r_{0}-d\right) / d$ and $N+1-m d \geqslant 1$. Thus

$$
\#\left(\mathfrak{p}^{a+1} / \mathfrak{p}^{r}\right)^{N+1} \leqslant \# \mathbf{F}_{\mathfrak{p}}^{(N+1)\left(r-\left(r-r_{0}\right) / d\right)} \leqslant \# \mathbf{F}_{\mathfrak{p}}^{r(N+1-m-1 / d)+(N+1) r_{0} / d} .
$$

Dividing by $\# \mathbf{F}_{\mathfrak{p}}^{r \operatorname{dim} W}$ and using the previous lemma, we get that

$$
\begin{aligned}
\frac{\#\left\{x \in\left(\mathcal{O}_{\mathfrak{p}} / \mathfrak{p}^{r}\right)^{N+1} \mid f(x)=0 \text { in }\left(\mathcal{O}_{\mathfrak{p}} / \mathfrak{p}^{r}\right)^{m}\right\}}{\left(\# \mathbf{F}_{\mathfrak{p}}\right)^{r \operatorname{dim} W}} \\
=\left(1-\frac{1}{\# \mathbf{F}_{\mathfrak{p}}^{\delta}}\right)^{-1} \frac{N^{*}\left(\mathfrak{p}^{r}\right)}{\# \mathbf{F}_{\mathfrak{p}}^{r} \operatorname{dim} W}+O\left(\# \mathbf{F}_{\mathfrak{p}}^{-r / d}\right) .
\end{aligned}
$$

The equations $\left(f_{i}\right)_{1 \leqslant i \leqslant m}$ define an isomorphism

$$
\omega_{V}^{-1} \stackrel{\sim}{\longrightarrow} \mathcal{O}_{V}(\delta)
$$

Therefore, for any place $v$ of $F$ the metric on $\mathcal{O}_{V}(\delta)$ induced by the monomials of degree $\delta$ defines a metric $\|\cdot\|_{v}$ on $\omega_{V}^{-1}$. The height $\mathbf{H}$ defined by the corresponding metrized line bundle $\left(\omega_{V}^{-1},\left(\|\cdot\|_{v}\right)_{v \in \operatorname{Val}(F)}\right)$ verifies

$$
\forall x \in V(F), \quad \mathbf{H}(x)=\prod_{v \in \operatorname{Val}(F)} \sup _{0 \leqslant i \leqslant N}\left(\left|x_{i}\right|_{v}\right)^{\delta} .
$$

Corollary 3.5. With notations as in Proposition 3.1 one has for any finite place $\mathfrak{p}$ of $F$

$$
\boldsymbol{\omega}_{\mathbf{H}, \mathfrak{p}}\left(V\left(F_{\mathfrak{p}}\right)\right)=\frac{1-\# \mathbf{F}_{\mathfrak{p}}^{-\delta}}{1-\# \mathbf{F}_{\mathfrak{p}}^{-1}} \lim _{r \rightarrow+\infty} \frac{\#\left\{x \in\left(\mathcal{O}_{\mathfrak{p}} / \mathfrak{p}^{r}\right)^{N+1} \mid f(x)=0 \operatorname{in}\left(\mathcal{O}_{\mathfrak{p}} / \mathfrak{p}^{r}\right)^{m}\right\}}{\# \mathbf{F}_{\mathfrak{p}}^{r} \operatorname{dim} W} .
$$


Proof. This follows from Proposition 3.1 and [Pe1, Lemme 5.4.6].

Remark 3.6. In particular, a factor $1 / 3$ is erroneously introduced in the first formula giving the constant $C_{\mathrm{HB}}(V)$ on page 148 in [Pe1] (see also [S-D, p. 374]) and therefore a factor 3 is missing in Proposition 5.6.1 of [Pe1]. In fact, if $V$ is the cubic surface defined by the equation

$$
X_{0}^{3}+X_{1}^{3}+X_{2}^{3}=k X_{3}^{3}
$$

with $k=2$ or 3 , one gets the equality

$$
\mathfrak{S}_{k}=\alpha(V) \beta(V) \tau_{\mathbf{H}}(V),
$$

where $\mathfrak{S}_{k}$ is the constant defined by Heath-Brown in [H-B]. Therefore, the numerical experiments made by Heath-Brown are compatible with the constant $\boldsymbol{\theta}_{\mathbf{H}}(V)$ as in Definition 2.6, and the Remark 2.3.2 in [Pe1] has to be corrected accordingly.

\section{Points on Cubics over $\mathbf{F}_{p}$}

We now describe explicitly the cardinal of $V\left(\mathbf{F}_{p}\right)$ when $V$ is the diagonal cubic surface given by the equation

$$
X_{0}^{3}+q^{2} X_{1}^{3}+q r X_{2}^{3}+r^{2} X_{3}^{3}=0,
$$

where $q, r \in \mathbf{Z}_{>1}$ are squarefree and coprime. We put $K_{1}=\mathbf{Q}\left(q^{1 / 3}\right), K_{2}=\mathbf{Q}\left(r^{1 / 3}\right)$, and $K_{3}=\mathbf{Q}\left((q r)^{1 / 3}\right)$ and consider

$$
\nu_{q, r}(p)=\#\left\{i \mid p \text { is totally split in } K_{i}\right\} .
$$

Proposition 4.1. If $p \nmid 3 q r$, then

$$
\frac{\# V\left(\mathbf{F}_{p}\right)}{p^{2}}=\left\{\begin{array}{l}
1+\frac{1}{p}+\frac{1}{p^{2}} \quad \text { if } p \equiv 2 \bmod 3 \\
1+\frac{3 \nu_{q, r}(p)-2}{p}+\frac{1}{p^{2}} \quad \text { otherwise }
\end{array}\right.
$$

Remark 4.2. By a result of Weil (see [Ma2, Theorem 23.1]),

$$
\frac{\# V\left(\mathbf{F}_{p}\right)}{p^{2}}=1+\operatorname{Tr}\left(\operatorname{Fr}_{p} \mid \operatorname{Pic}(\bar{V})\right) p+p^{2},
$$

and the only difficulty is to determine $\operatorname{Tr}\left(\operatorname{Fr}_{p} \mid \operatorname{Pic}(\bar{V})\right)$. We have chosen to avoid this computation by using a general formula valid for diagonal hypersurfaces.

Remark 4.3. If $p \equiv 1 \bmod 3$, then $\mathbf{F}_{p}$ contains the cubic roots of 1 . Therefore $\nu_{q, r}(p)$ is either 3,1 or 0 . In other words, the possible values in this case are

$$
1+\frac{7}{p}+\frac{1}{p^{2}}, \quad 1+\frac{1}{p}+\frac{1}{p^{2}}, \quad 1-\frac{2}{p}+\frac{1}{p^{2}} .
$$

Proof. Let $N(p)$ be the number of solutions of (4) in $\left(\mathbf{F}_{p}\right)^{4}$. By [IR] $\S 8.7$, Theorem $5]$, we have the formula

$$
N(p)=p^{3}+\sum \bar{\chi}_{1}(1) \bar{\chi}_{2}\left(q^{2}\right) \bar{\chi}_{3}\left(r^{2}\right) \bar{\chi}_{4}(q r) J_{0}\left(\chi_{1}, \ldots, \chi_{4}\right),
$$


where the sum is taken over the quadruples of nontrivial cubic characters $\chi_{1}, \chi_{2}$, $\chi_{3}, \chi_{4}$ from $\mathbf{F}_{p}^{*}$ to $\mathbf{C}^{*}$ such that $\chi_{1} \chi_{2} \chi_{3} \chi_{4}$ is the trivial character and where

$$
J_{0}\left(\chi_{1}, \ldots, \chi_{4}\right)=\sum_{t_{1}+\cdots+t_{4}=0} \prod_{i=1}^{4} \chi_{i}\left(t_{i}\right),
$$

with the convention $\chi_{i}(0)=0$. If $p \equiv 2 \bmod 3$, then there are no nontrivial characters, and we get that

$$
\# V\left(\mathbf{F}_{p}\right)=\frac{N(p)-1}{p-1}=1+p+p^{2} .
$$

Otherwise, there are exactly two nontrivial characters which are conjugate and will be denoted by $\chi$ and $\bar{\chi}$. By IR, $\S 8.5$, Theorem 4], we have

$$
\left|J_{0}(\chi, \chi, \bar{\chi}, \bar{\chi})\right|=p(p-1) .
$$

But, by definition, this complex number may be written as

$$
\begin{aligned}
J_{0}(\chi, \chi, \bar{\chi}, \bar{\chi}) & =\sum_{t_{1}+\cdots+t_{4}=0} \chi\left(t_{1} t_{2}\right) \bar{\chi}\left(t_{3} t_{4}\right) \\
& =\sum_{a \in \mathbf{F}_{p}}\left|\sum_{t_{1}+t_{2}=a} \chi\left(t_{1} t_{2}\right)\right|^{2}
\end{aligned}
$$

and is a positive real number. Finally we get

$$
N(p)=p^{3}+p(p-1) \sum \chi_{1}\left(q^{2}\right) \chi_{2}\left(r^{2}\right) \chi_{3}(q r),
$$

where the sum is taken over all nontrivial cubic characters such that $\chi_{1} \chi_{2} \chi_{3}$ is nontrivial. This sum may be written as

$$
\begin{aligned}
\sum \chi_{1}\left(q^{2}\right) \chi_{2}\left(r^{2}\right) \chi_{3}(q r)= & \chi\left(q^{2}\right) \chi\left(r^{2}\right) \bar{\chi}(q r)+\chi\left(q^{2}\right) \bar{\chi}\left(r^{2}\right) \chi(q r) \\
& +\bar{\chi}\left(q^{2}\right) \chi\left(r^{2}\right) \chi(r q)+\chi\left(q^{2}\right) \bar{\chi}\left(r^{2}\right) \bar{\chi}(q r) \\
& +\bar{\chi}\left(q^{2}\right) \chi\left(r^{2}\right) \bar{\chi}(q r)+\bar{\chi}\left(q^{2}\right) \bar{\chi}\left(r^{2}\right) \chi(q r) \\
= & \chi(q)+\bar{\chi}(q)+\chi(r)+\bar{\chi}(r)+\chi(q r)+\bar{\chi}(q r) .
\end{aligned}
$$

Observe that for any integer $n$ prime to $p$ one has

$$
\chi(n)+\bar{\chi}(n)= \begin{cases}-1 & \text { if } p \text { is not split in } \mathbf{Q}\left(n^{1 / 3}\right), \\ 2 & \text { otherwise. }\end{cases}
$$

Lemma 4.4. With notation as above, if $p \equiv 2 \bmod 3, p \neq 2$, and $p \mid q r$, then

$$
\frac{N^{*}\left(p^{t}\right)}{p^{3 t}}=1-\frac{1}{p} \quad \text { if } t>0 .
$$

Proof. We may assume that $p \mid r$. Let $x=\left(x_{0}, x_{1}, x_{2}, x_{3}\right)$ be a solution of (4) in $\left(\mathbf{Z} / p^{t} \mathbf{Z}\right)^{4}-\left(p \mathbf{Z} / p^{t} \mathbf{Z}\right)^{4}$. If $p \mid x_{1}$, then by the equation $p \mid x_{0}$ and then $x \in(p)^{4}$, which gives a contradiction. Since the group of invertible elements in $\mathbf{Z} / p^{t} \mathbf{Z}$ is isomorphic 
to $\mathbf{Z} / p^{t-1}(p-1) \mathbf{Z}$, any element in this group has a unique cubic root. Therefore, the set of solutions is parametrized by the $\left(x_{1}, x_{2}, x_{3}\right) \in \mathbf{Z} / p^{t} \mathbf{Z}$ such that $p \nmid x_{1}$.

Lemma 4.5. With notations as above, if $q \equiv \pm r \bmod 9$ and $3 \chi q r$, then the possible values for $N^{*}\left(3^{2}\right) / 3^{6}$ are given by the following table.

\begin{tabular}{|c|c|c|c|}
\hline$q, r \bmod 9$ & \pm 1 & \pm 2 & \pm 4 \\
\hline$N^{*}\left(3^{2}\right) / 3^{6}$ & 2 & $2^{2} / 3$ & $2 / 3$ \\
\hline
\end{tabular}

Proof. Up to multiplication by units, the equation in this case may be written over $\mathbf{Q}_{3}$ as

$$
X^{3}+q^{2} Y^{3}+q^{2} Z^{3}+q^{2} T^{3}=0,
$$

which is equivalent to

$$
X^{3}+Y^{3}+Z^{3}+q T^{3}=0
$$

and the result follows from $[\mathrm{H}-\mathrm{B}]$ or a direct computation.

\section{Convergence Factors AND RESidues}

As in Heath-Brown $[\mathrm{H}-\mathrm{B}$, for the explicit computation of the constant we need a family of convergence factors related to zeta functions of cubic extensions of $\mathbf{Q}$. If $V$ is defined by (4), it follows from [CTKS, p. 12] that $t=\operatorname{rk} \operatorname{Pic}(V)=1$.

Proposition 5.1. If $V$ is the diagonal cubic given by the equation (4) and $K_{i}$ are the fields defined in the previous paragraph, then the measure $\boldsymbol{\omega}_{\mathbf{H}}$ coincides with the measure

$$
\lim _{s \rightarrow 1}(s-1) \frac{\prod_{i=1}^{3} \zeta_{K_{i}}(s)}{\zeta_{\mathbf{Q}}(s)^{2}} \prod_{v \in \operatorname{Val}(\mathbf{Q})} \lambda_{v}^{\prime} \boldsymbol{\omega}_{\mathbf{H}, v}
$$

where

$$
\lambda_{p}^{\prime}=\frac{\prod_{i=1}^{3} \prod_{\left\{\mathfrak{P} \in \operatorname{Val}\left(K_{i}\right)|\mathfrak{P}| p\right\}}\left(1-\# \mathbf{F}_{\mathfrak{P}}^{-1}\right)}{\left(1-p^{-1}\right)^{2}} \quad \text { if } p \text { is a prime number and } \lambda_{\mathbf{R}}^{\prime}=1 .
$$

Remark 5.2. If $p$ does not divide $3 q r$, we can use the term $\lambda_{p}^{\prime} \boldsymbol{\omega}_{\mathbf{H}, p}\left(V\left(\mathbf{Q}_{p}\right)\right)$ which by Lemma 3.2, Lemma 3.4 and Lemma 5.4.6 in [Pe1] coincides with $\lambda_{p}^{\prime} \# V\left(\mathbf{F}_{p}\right) / p^{2}$ (see also [Pe1, Lemme 2.2.1]) and by Proposition 4.1 is equal to

$$
\begin{array}{ll}
\left(1-\frac{1}{p}\right)^{7}\left(1+\frac{7}{p}+\frac{1}{p^{2}}\right) & \text { if } p \equiv 1 \bmod 3 \text { and } \nu_{q, r}(p)=3, \\
\left(1-\frac{1}{p}\right)\left(1-\frac{1}{p^{3}}\right)^{2}\left(1+\frac{1}{p}+\frac{1}{p^{2}}\right) & \text { if } p \equiv 1 \bmod 3 \text { and } \nu_{q, r}(p)=1, \\
\left(1-\frac{1}{p}\right)\left(1+\frac{1}{p}+\frac{1}{p^{2}}\right)^{3}\left(1-\frac{2}{p}+\frac{1}{p^{2}}\right) & \text { if } p \equiv 1 \bmod 3 \text { and } \nu_{q, r}(p)=0, \\
\left(1-\frac{1}{p}\right)\left(1-\frac{1}{p^{2}}\right)^{3}\left(1+\frac{1}{p}+\frac{1}{p^{2}}\right) & \text { if } p \equiv 2 \bmod 3,
\end{array}
$$


and the good places yield a product $C_{1} C_{2} C_{3}$ where

$$
\begin{aligned}
& C_{1}=\prod_{\substack{p \nmid 3 q r \\
p \equiv 1 \bmod 3 \\
\nu_{q, r}(p)=3}}\left(1-\frac{1}{p}\right)^{7}\left(1+\frac{7}{p}+\frac{1}{p^{2}}\right), \\
& C_{2}=\prod_{\substack{p \nmid 3 q r \\
p \equiv 1 \bmod 3 \\
\nu_{q, r}(p) \neq 3}}\left(1-\frac{1}{p^{3}}\right)^{3}, \\
& C_{3}=\prod_{\substack{p \nmid 3 q r \\
p \equiv 2 \bmod 3}}\left(1-\frac{1}{p^{3}}\right)\left(1-\frac{1}{p^{2}}\right)^{3} .
\end{aligned}
$$

Proof. It follows from the proof of Proposition 4.1 that, if $p \nmid 3 q r$,

$$
\frac{\# V\left(\mathbf{F}_{p}\right)}{p^{2}}=1+\frac{a(p)}{p}+\frac{1}{p^{2}}
$$

where

$$
a(p)=\left\{\begin{array}{l}
1 \text { if } p \equiv 2 \bmod (3), \\
1+\chi(q)+\bar{\chi}(q)+\chi(r)+\bar{\chi}(r)+\chi(q r)+\bar{\chi}(q r) \text { otherwise }
\end{array}\right.
$$

where $\chi$ is a nontrivial cubic character of $\mathbf{F}_{p}$, if $p \equiv 1 \bmod 3$. It follows from a theorem of Weil (see [Ma2, Theorem 23.1]) that, outside a finite set of places,

$$
\# V\left(\mathbf{F}_{p}\right)=1+\operatorname{Tr}\left(\operatorname{Fr}_{p} \mid \operatorname{Pic}(\bar{V})\right) p+p^{2} .
$$

Since the action of the Galois group on the Picard group splits over a finite extension of $k$, the eigenvalues of the Frobenius map for this action are roots of unity. Therefore we get that

$$
L_{p}(s, \operatorname{Pic}(\bar{V}))^{-1}=1-\frac{a(p)}{p^{s}}+R_{p}\left(\frac{1}{p^{s}}\right),
$$

where the $R_{p}$ are polynomials of order at least 2 with uniformly bounded coefficients with respect to $p$.

But for any cubefree integer $n$, the zeta function of $\mathbf{Q}\left(n^{1 / 3}\right)$ may be written as an Euler product with local terms $\zeta_{\mathbf{Q}\left(n^{1 / 3}\right), p}(s)$ which for $p \nmid 3 n$ are given by

$$
\zeta_{\mathbf{Q}\left(n^{1 / 3}\right), p}(s)^{-1}= \begin{cases}\left(1-\frac{1}{p^{s}}\right)\left(1-\frac{1}{p^{2 s}}\right) & \text { if } p \equiv 2 \bmod 3, \\ \left(1-\frac{1}{p^{s}}\right)\left(1-\frac{\chi(n)}{p^{s}}\right)\left(1-\frac{\bar{\chi}(n)}{p^{s}}\right) & \text { if } p \equiv 1 \bmod 3 .\end{cases}
$$

Thus, by (6), for almost all places, the local terms of the zeta functions verify

$$
\left(\zeta_{\mathbf{Q}, p}(s) \prod_{i=1}^{3} \frac{\zeta_{K_{i}, p}(s)}{\zeta_{\mathbf{Q}, p}(s)}\right)^{-1}=1-\frac{a(p)}{p^{s}}+Q_{p}\left(\frac{1}{p^{s}}\right)
$$

where the $Q_{p}$ are polynomials of order at least 2 with bounded coefficients. Using (5) we get that the product of measures given in the proposition defines a Borel 
measure on the adelic points of $V$ and by (7) that the Euler product defining the quotient

$$
L_{S}(s, \operatorname{Pic}(\bar{V})) / \frac{\prod_{i=1}^{3} \zeta_{K_{i}}(s)}{\zeta_{\mathbf{Q}}(s)^{2}}
$$

converges absolutely in $s=1$. Since $d_{\mathbf{Q}}=1$, we get that

$$
\begin{aligned}
\boldsymbol{\omega}_{\mathbf{H}} & =\lim _{s \rightarrow 1}\left((s-1) L_{S}(s, \operatorname{Pic}(\bar{V}))\right) \prod_{v \in \operatorname{Val}(\mathbf{Q})} \lambda_{v}^{-1} \boldsymbol{\omega}_{\mathbf{H}, v} \\
& =\lim _{s \rightarrow 1}\left((s-1) L_{S}(s, \operatorname{Pic}(\bar{V}))\right) \prod_{v \in \operatorname{Val}(\mathbf{Q})} \frac{\lambda_{v}^{-1}}{\lambda_{v}^{\prime}} \prod_{v \in \operatorname{Val}(\mathbf{Q})} \lambda_{v}^{\prime} \boldsymbol{\omega}_{\mathbf{H}, v} \\
& =\lim _{s \rightarrow 1}\left((s-1) L_{S}(s, \operatorname{Pic}(\bar{V}))\right) \lim _{s \rightarrow 1} \frac{\left(\prod_{i=1}^{3} \zeta_{K_{i}}(s)\right) / \zeta_{\mathbf{Q}}(s)^{2}}{L_{S}(s, \operatorname{Pic}(\bar{V}))} \prod_{v \in \operatorname{Val}(\mathbf{Q})} \lambda_{v}^{\prime} \boldsymbol{\omega}_{\mathbf{H}, v} \\
& =\lim _{s \rightarrow 1}(s-1) \frac{\prod_{i=1}^{3} \zeta_{K_{i}}(s)}{\zeta_{\mathbf{Q}}(s)^{2}} \prod_{v \in \operatorname{Val}(\mathbf{Q})} \lambda_{v}^{\prime} \boldsymbol{\omega}_{\mathbf{H}, v} .
\end{aligned}
$$

\section{Brauer-Manin obstruction to Weak approximation}

In this paragraph, using the work of Colliot-Thélène, Kanevsky, and Sansuc [CTKS], we shall compute the quotient

$$
\boldsymbol{\omega}_{\mathbf{H}}\left(V\left(A_{\mathbf{Q}}\right)^{\mathrm{Br}}\right) / \boldsymbol{\omega}_{\mathbf{H}}\left(V\left(A_{\mathbf{Q}}\right)\right)
$$

when $V$ is the diagonal cubic defined by equation (4) and $V\left(A_{\mathbf{Q}}\right) \neq \emptyset$.

In the following we assume that $q$ and $r$ are distinct prime numbers such that 3 \ qr. It follows from [CTKS, p. 28] that $V\left(A_{\mathbf{Q}}\right) \neq \emptyset$ if and only if the following condition is satisfied:

$$
\left(q \equiv 2 \bmod 3 \quad \text { or } \quad r \in \mathbf{F}_{q}^{* 3}\right) \quad \text { and } \quad\left(r \equiv 2 \bmod 3 \quad \text { or } \quad q \in \mathbf{F}_{r}^{* 3}\right) .
$$

Proposition 6.1. Under these assumptions, the value for the quotient (8) depends only on the classes of $p$ and $q$ modulo 9. These values are given in the following table.

\begin{tabular}{|c||c|c|c|c|c|c|}
\hline$r \Upsilon q$ & 1 & 2 & 4 & 5 & 7 & 8 \\
\hline \hline 1 & 1 & 1 & 1 & 1 & 1 & 1 \\
\hline 2 & 1 & $1 / 3$ & 0 & 0 & $1 / 3$ & 1 \\
\hline 4 & 1 & 0 & $1 / 3$ & $1 / 3$ & 0 & 1 \\
\hline 5 & 1 & 0 & $1 / 3$ & $1 / 3$ & 0 & 1 \\
\hline 7 & 1 & $1 / 3$ & 0 & 0 & $1 / 3$ & 1 \\
\hline 8 & 1 & 1 & 1 & 1 & 1 & 1 \\
\hline
\end{tabular}


Proof. Let $j$ be a primitive third root of unity, $k=\mathbf{Q}(j)$ and $K=k\left(q^{1 / 3}, r^{1 / 3}\right)$. We have the following diagram of fields

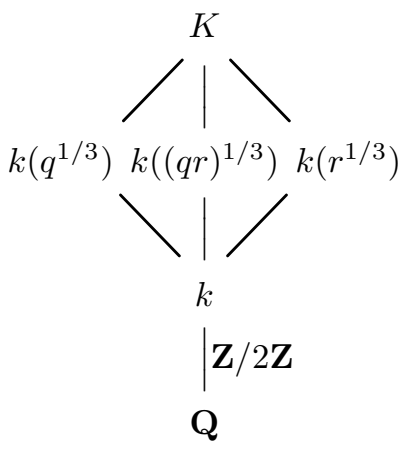

and the group $G=\operatorname{Gal}(K / \mathbf{Q})$ may be described as the semidirect product $(\mathbf{Z} / 3 \mathbf{Z})^{2} \rtimes$ $\mathbf{Z} / 2 \mathbf{Z}$ where $\mathbf{Z} / 2 \mathbf{Z}$ acts by - Id on $(\mathbf{Z} / 3 \mathbf{Z})^{2}$. By [CTKS Proposition 1, p. 7], we have that

$$
\operatorname{Br}\left(V \times{ }_{\mathbf{Q}} k\right) / \operatorname{Br}(k) \stackrel{\sim}{\longrightarrow} H^{1}(k, \operatorname{Pic}(\bar{V})) \stackrel{\sim}{\longrightarrow} \mathbf{Z} / 3 \mathbf{Z} .
$$

But the Hochschild-Serre spectral sequence gives an exact sequence

$$
\begin{aligned}
0 \rightarrow H^{1}\left(\mathbf{Z} / 2 \mathbf{Z},(\operatorname{Pic}(\bar{V}))^{(\mathbf{Z} / 3 \mathbf{Z})^{2}}\right) \rightarrow H^{1}(\mathbf{Q}, \operatorname{Pic}(\bar{V})) \\
\\
\rightarrow\left(H^{1}(k, \operatorname{Pic}(\bar{V}))\right)^{\mathbf{Z} / 2 \mathbf{Z}} \rightarrow H^{2}\left(\mathbf{Z} / 2 \mathbf{Z},(\operatorname{Pic}(\bar{V}))^{(\mathbf{Z} / 3 \mathbf{Z})^{2}}\right) .
\end{aligned}
$$

By [CTKS, p. 12], $\operatorname{Pic}(\bar{V})^{\operatorname{Gal}(\bar{k} / k)}=\mathbf{Z}$ and, since a map from a group killed by 3 to one killed by 2 is trivial, we obtain an isomorphism

$$
H^{1}(\mathbf{Q}, \operatorname{Pic}(\bar{V})) \stackrel{\sim}{\longrightarrow} H^{1}(k, \operatorname{Pic}(\bar{V}))^{\mathbf{Z} / 2 \mathbf{Z}} .
$$

By the proof of [CTKS, Lemme 3, p. 13], we have isomorphisms

$$
H^{1}(k, \operatorname{Pic}(\bar{V})) \stackrel{\sim}{\longrightarrow} H^{1}(\mathbf{Z} / 3 \mathbf{Z}, \mathbf{Z} \oplus Q) \stackrel{\sim}{\longrightarrow} H^{1}(\mathbf{Z} / 3 \mathbf{Z}, Q),
$$

where $\mathrm{Q}$ is the $\mathbf{Z} / 3 \mathbf{Z}$-module defined by the exact sequence

$$
0 \rightarrow \mathbf{Z} \stackrel{N}{\rightarrow} \mathbf{Z}[\mathbf{Z} / 3 \mathbf{Z}] \rightarrow Q \rightarrow 0 .
$$

But for any cyclic group $C$ and any $C$-module $M$ there is canonical injection

$$
H^{1}(C, M) \rightarrow \operatorname{Hom}\left(C, M_{C}\right),
$$

where $M_{C}$ denotes the group of coinvariants and which sends the class of a cocycle $\gamma$ onto the induced morphism $\bar{\gamma}$ from $C$ to $M_{C}$, so that the diagram

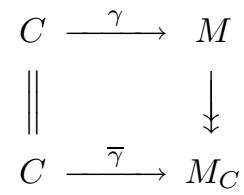

commutes. If $C$ is a normal subgroup of a group $H$, then the above morphism is compatible with the natural actions of $H / C$ on $H^{1}(C, M)$ and $\operatorname{Hom}\left(C, M_{C}\right)$. It follows from [CTKS, Lemme 3] that $\mathbf{Z} / 2 \mathbf{Z}$ acts by - Id on the torsion part of $Q_{\mathbf{Z} / 3 \mathbf{Z}}$ and by the above description of $G$ as a semidirect product, $\mathbf{Z} / 2 \mathbf{Z}$ acts by -1 on $\mathbf{Z} / 3 \mathbf{Z}$. Therefore the action of $\mathbf{Z} / 2 \mathbf{Z}$ on $H^{1}(\mathbf{Z} / 3 \mathbf{Z}, Q)$ is trivial and

$$
H^{1}(\mathbf{Q}, \operatorname{Pic}(\bar{V}))=\mathbf{Z} / 3 \mathbf{Z} \text {. }
$$


For any prime $p$, the canonical pairing

$$
\begin{aligned}
\operatorname{Br}(V) \times V\left(\mathbf{Q}_{p}\right) & \rightarrow \mathbf{Q} / \mathbf{Z} \\
([A], P) & \mapsto \operatorname{inv}_{p}(A(P))
\end{aligned}
$$

defines an equivalence relation on $V\left(\mathbf{Q}_{p}\right)$ which is also denoted by $\mathrm{Br}$. If $V$ has good reduction in $p$, then by [CT1, Theorem A (iii)] the rational equivalence on $V\left(\mathbf{Q}_{p}\right)$ is trivial and therefore the same is true of the Brauer equivalence. The condition (9) implies that if $p \mid q r$, then $V$ is rational over $\mathbf{Q}_{p}$ (see [CTKS, Lemme 8, p. 41]) and $\# V\left(\mathbf{Q}_{p}\right) / \mathrm{Br}=1$. Using the same type of argument, we get that $\# V\left(\mathbf{Q}_{3}\right) / \mathrm{Br}=1$ if $q, r$ or $q r$ is a cube modulo 9 , that is one of them is 1 or -1 modulo 9. On the other hand, it follows from CTKS, §5, p. 41] that this cardinal is 3 at 3 otherwise. This explains the dichotomy between integral and nonintegral values in the table.

We have to find an element $A \in \operatorname{Br}(V)$ whose class spans $\operatorname{Br}(V) / \operatorname{Br}(\mathbf{Q})$. Then the Brauer equivalence and the Brauer obstruction may be computed in terms of the functions

$$
\begin{aligned}
i_{p}: V\left(\mathbf{Q}_{p}\right) & \rightarrow \mathbf{Z} / 3 \mathbf{Z} \\
P & \mapsto \operatorname{inv}_{p}(A(P)) .
\end{aligned}
$$

This is an intricate procedure. It is described in detail on pages 19-72 and summarized in an algorithm on pages 73-79 in [CTKS], which we are going to follow.

Let us first assume that $q, r$ or $q r$ is a cube modulo 9 . Then all functions $i_{p}$ are constant and $A$ may be chosen so that the value $i_{p}$ of the function $i_{p}$ is trivial except when $p$ equals $3, q$, or $r$. It remains to compute the constant values $i_{3}, i_{q}$, and $i_{r}$ for such an $A$. To this end, we use the additive norm rest symbols $[., .]_{p}$ from $k_{v}^{*}$ to $\mathbf{Z} / 3 \mathbf{Z}$, for $v$ in $\operatorname{Val}(k)$, dividing $p$ (see [CTKS p. 77]). They are biadditive, anticommutative, and verify the relations

$$
\begin{aligned}
& {[j, p]_{p}=\frac{p-1}{3} \quad \text { if } p \equiv 1 \bmod 3} \\
& {[j, p]_{p}=-\frac{p^{2}-1}{3} \quad \text { if } p \equiv 2 \bmod 3 .}
\end{aligned}
$$

If $r$ is a cube in $\mathbf{Q}_{3}$, we get that $A$ may be chosen so that

$$
i_{3}=0, \quad i_{q}=0, \quad i_{r}=[j, r]_{r} .
$$

Since $r \equiv \pm 1 \bmod 9$, we have that $i_{r}=0$ and the Brauer-Manin obstruction is trivial. If $q$ is a cube modulo 9 , the result is similar and the quotient (8) equals 1 .

If $q r$ is a cube modulo 9 but $q$ and $r$ are not, then the situation is similar to the one considered in [CTKS, Proposition 5, p. 67-69], and one writes the equation as

$$
X^{3}+q Y^{3}+q^{2} r Z^{3}+q^{4} r^{2} T^{3}=0
$$

and, by [CTKS, §8], $A$ may be chosen so that

$$
i_{3}=0, \quad i_{q}=0 \quad \text { and } \quad i_{r}=[j, r]_{r} .
$$

The values of $i_{r}$ are given by the following table (see [CTKS, p. 78]).

\begin{tabular}{|l|l|l|l|l|}
\hline$r \bmod 9$ & 2 & 4 & 5 & 7 \\
\hline$[j, r]_{r}$ & 2 & 1 & 1 & 2 \\
\hline
\end{tabular}

In this case there is a Brauer-Manin obstruction to the Hasse principle and the quotient (8) is zero. 
If none of the $q, r$ and $q r$ is a cube in $\mathbf{Q}_{3}$, then to complete the proof of Proposition 6.1 we have only to prove that each class in $V\left(\mathbf{Q}_{3}\right)$ for the Brauer equivalence has the same volume for $\boldsymbol{\omega}_{\mathbf{H}, 3}$.

Up to permutation and change of sign, we may assume that $q \equiv r \equiv 2$ modulo 9 or $q \equiv r \equiv 4$ modulo 9 . Therefore the equation modulo 9 may be written as

$$
X^{3}+4 Y^{3}+4 Z^{3}+4 T^{3}=0
$$

or

$$
X^{3}+16 Y^{3}+16 Z^{3}+16 T^{3}=0 .
$$

Therefore on $\mathbf{Q}_{3}$ the equation is equivalent to

$$
X^{3}+Y^{3}+Z^{3}+2 T^{3}=0
$$

or

$$
X^{3}+Y^{3}+Z^{3}+4 T^{3}=0 .
$$

A direct computation modulo 9 shows that exactly one of the three first coordinates has to be divisible by 3 . But in the first case, it follows from $\overline{\mathrm{H}-\mathrm{B}}$, proof of Theorem 1] that the classes for the Brauer equivalence are determined by the coordinate which vanishes modulo 3 . By a symmetry argument the volumes of the equivalence classes are the same.

By [CTKS, p. 49], there is a choice of $A$ so that the induced map $i_{3}$ over $V\left(\mathbf{Q}_{3}(j)\right)$ is given as

$$
i_{3}(x, y, z, t)=-[x+j y, \nu]_{3},
$$

where $\nu$ is the coefficient of $T^{3}$. Using the commutative diagram

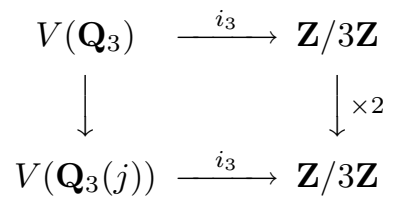

we get that the invariants for the second equation may be described, after reduction modulo 9 , as the double of the ones for the first and we get a similar description of $V\left(\mathbf{Q}_{3}\right) /$ Br. This implies the result in this case.

\section{Numerical Tests}

The numerical tests for the number of points with bounded heights have been conducted using an efficient program of Dan Bernstein [Be]. We considered the following cubic surfaces

$$
\begin{aligned}
X_{0}^{3}+17^{2} X_{1}^{3}+17 \times 53 X_{2}^{3}+53^{2} X_{3}^{3} & =0, \\
X_{0}^{3}+71^{2} X_{1}^{3}+71 \times 53 X_{2}^{3}+53^{2} X_{3}^{3} & =0, \\
X_{0}^{3}+5^{2} X_{1}^{3}+23 \times 5 X_{2}^{3}+23^{2} X_{3}^{3} & =0, \\
X_{0}^{3}+11^{2} X_{1}^{3}+29 \times 11 X_{2}^{3}+29^{2} X_{3}^{3} & =0 .
\end{aligned}
$$

One can take for the open set $U$ the whole surface $V$, as there are no rational points on the exceptional curves. The graphs of $n_{U, \mathbf{H}}$ are presented below. 

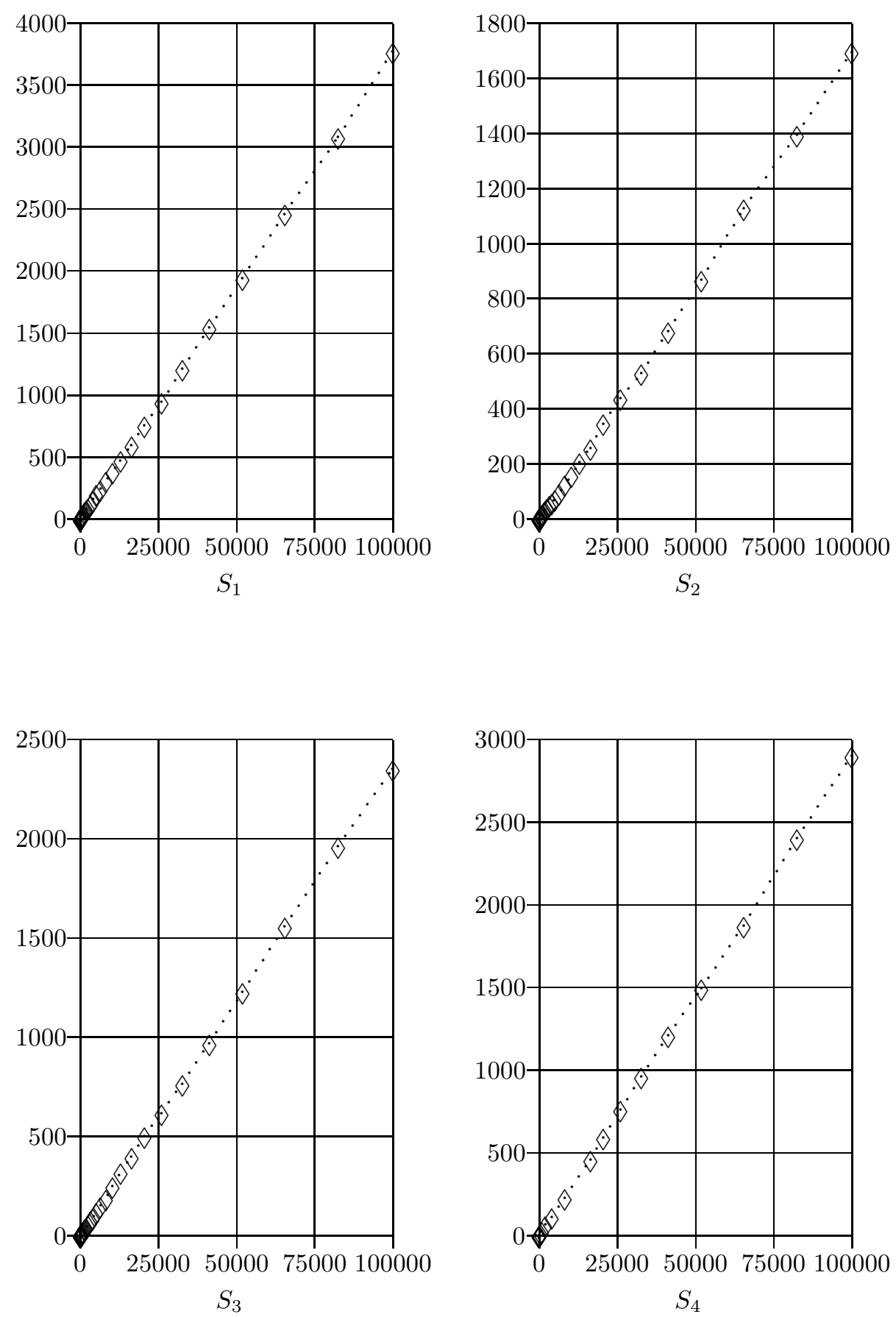

Let us sum up the description of the theoretical constant. Let $V$ be a diagonal cubic over $\mathbf{Q}$ defined by the equation (4) with $q$ and $r$ distinct prime numbers such that $3 \times q r$ and such that the condition (9) is satisfied. 
TABLE 1.

\begin{tabular}{|l|l|l|l|l|}
\hline Surface & $S_{1}$ & $S_{2}$ & $S_{3}$ & $S_{4}$ \\
\hline$H$ & 99999 & 99999 & 99999 & 99999 \\
\hline$n_{U, \mathbf{H}}(H)$ & 3773 & 1696 & 2353 & 2904 \\
\hline$C_{\mathrm{Br}}$ & 1 & 1 & $1 / 3$ & $1 / 3$ \\
\hline$H^{1}(\mathbf{Q}, \operatorname{Pic}(\bar{V}))$ & 3 & 3 & 3 & 3 \\
\hline$\zeta_{\mathbf{Q}\left(q^{1 / 3}\right)}^{*}(1)$ & 1.4680 & 1.8172 & 1.1637 & 1.2284 \\
\hline$\zeta_{\mathbf{Q}\left(r^{1 / 3}\right)}(1)$ & 1.8172 & 2.2035 & 1.1879 & 1.6792 \\
\hline$\zeta_{\mathbf{Q}\left((q r)^{1 / 3}\right)}^{*}(1)$ & 1.9342 & 1.9925 & 1.0865 & 1.0543 \\
\hline$\lambda_{3}^{\prime} \boldsymbol{\omega}_{\mathbf{H}}\left(V\left(\mathbf{Q}_{3}\right)\right)$ & 0.5926 & 0.5926 & 0.6667 & 1.3333 \\
\hline$\lambda_{q}^{\prime} \boldsymbol{\omega}_{\mathbf{H}}\left(V\left(\mathbf{Q}_{q}\right)\right)$ & 0.9379 & 0.9808 & 0.7680 & 0.9016 \\
\hline$\lambda_{r}^{\prime} \boldsymbol{\omega}_{\mathbf{H}}\left(V\left(\mathbf{Q}_{r}\right)\right)$ & 0.9808 & 0.9857 & 0.9547 & 0.9644 \\
\hline$C_{1}$ & 0.9978 & 0.9989 & 0.9973 & 0.9812 \\
\hline$C_{2}$ & 0.9892 & 0.9892 & 0.9892 & 0.9893 \\
\hline$C_{3}$ & 0.3103 & 0.3072 & 0.3514 & 0.3158 \\
\hline $\boldsymbol{\omega}_{\mathbf{H}}(V(\mathbf{R}))$ & 0.0148 & 0.0042 & 0.0917 & 0.0389 \\
\hline $\boldsymbol{\theta}_{\mathbf{H}}(V)$ & 0.0382 & 0.0175 & 0.0234 & 0.0300 \\
\hline$n_{U, \mathbf{H}}(H) / \boldsymbol{\theta}_{\mathbf{H}}(V) H$ & 0.9882 & 0.9669 & 1.0077 & 0.9665 \\
\hline
\end{tabular}

By Proposition 5.1 the constant $\boldsymbol{\theta}_{\mathbf{H}}(V)$ may be written as the product

$$
\begin{aligned}
\frac{\boldsymbol{\omega}_{\mathbf{H}}\left(V\left(A_{\mathbf{Q}}\right)^{\mathrm{Br}}\right)}{\boldsymbol{\omega}_{\mathbf{H}}\left(V\left(A_{\mathbf{Q}}\right)\right)} \# H^{1}(\mathbf{Q}, & \operatorname{Pic}(\bar{V})) \\
& \times \prod_{i=1}^{3} \zeta_{K_{i}}^{*}(1) \prod_{p \mid 3 q r} \lambda_{p}^{\prime} \boldsymbol{\omega}_{\mathbf{H}, p}\left(V\left(\mathbf{Q}_{p}\right)\right) C_{1} C_{2} C_{3} \boldsymbol{\omega}_{\mathbf{H}, \mathbf{R}}(V(\mathbf{R})),
\end{aligned}
$$

where the first term which will be denoted by $C_{\mathrm{Br}}$ may be found in Proposition 6.1 the cardinal of $H^{1}(\mathbf{Q}, \operatorname{Pic}(\bar{V}))$ equals 3 , the residues of the zeta functions $\zeta_{K_{i}}^{*}(1)$ have been computed using Dirichlet's class number formula and the system PARI (see also [Co, Chapter 4]), $\lambda_{p}^{\prime}$ is defined in Proposition [5.1 the volumes at the bad places are given in Lemmata 4.4 and 4.5 and $C_{1}, C_{2}, C_{3}$ have been described as absolutely convergent Euler products (see Remark 5.2). The volume at the real place may be computed directly using the definition of the Leray form. The computations are summarized in Table 1 .

The new program of Bernstein allows us to increase the upper bound for the height of rational points on the cubic surfaces studied by Heath-Brown in [H-B]. 
These cubics are defined by the equations

$$
\begin{aligned}
& X_{0}^{3}+X_{1}^{3}+X_{2}^{3}+2 X_{3}^{3}=0 \\
& X_{0}^{3}+X_{1}^{3}+X_{2}^{3}+3 X_{3}^{3}=0 .
\end{aligned}
$$

The graphs of $n_{U, \mathbf{H}}$ are drawn below.
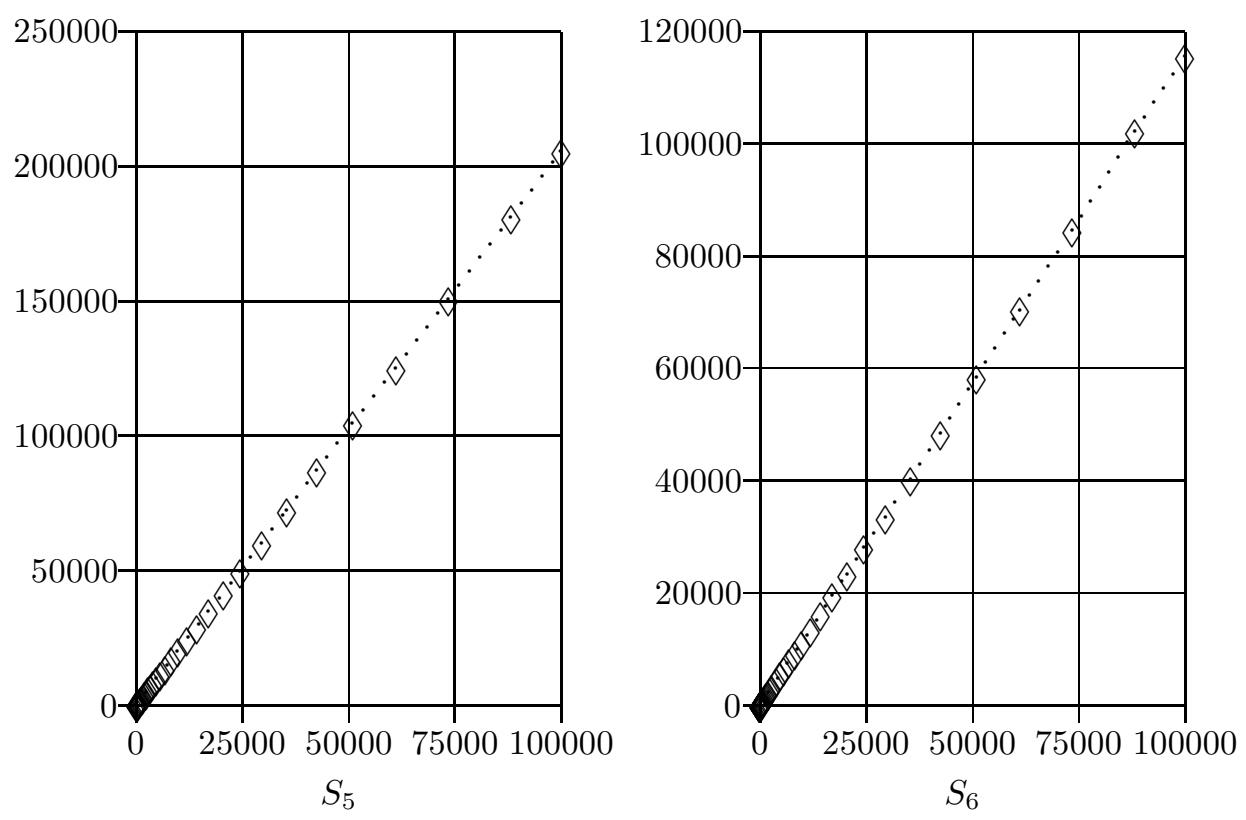

In this case, by Remark 3.6 and [H-B], the constant $\boldsymbol{\theta}_{\mathbf{H}}(V)$ may be written as the product

$$
\begin{aligned}
\frac{\boldsymbol{\omega}_{\mathbf{H}}\left(V\left(A_{\mathbf{Q}}\right)^{\mathrm{Br}}\right)}{\boldsymbol{\omega}_{\mathbf{H}}\left(V\left(A_{\mathbf{Q}}\right)\right)} \# H^{1}(\mathbf{Q}, & \operatorname{Pic}(\bar{V})) \\
& \times \zeta_{\mathbf{Q}\left(q^{1 / 3}\right)}^{*}(1)^{3} \prod_{p \mid 3 q} \lambda_{p}^{\prime} \boldsymbol{\omega}_{\mathbf{H}, p}\left(V\left(\mathbf{Q}_{p}\right)\right) C_{1} C_{2} C_{3} \boldsymbol{\omega}_{\mathbf{H}, \mathbf{R}}(V(\mathbf{R})),
\end{aligned}
$$

where the first factor is $1 / 3$ for these cubics, the second $3, q$ is the last coefficient in the equation, the local factors at the bad places are given by

$$
N^{*}\left(3^{2}\right)=\left\{\begin{array}{ll}
2^{2} 3^{5} & \text { if } q=2 \\
2^{3} 3^{4} & \text { if } q=3
\end{array} \quad N^{*}(4)=2^{6}-2^{4} \quad \text { if } \quad q=2,\right.
$$

and $C_{1}, C_{2}$ and $C_{3}$ are defined exactly as in Remark 5.2 The results are given in Table 2. Therefore, the numerical tests for these cubic surfaces are compatible with an asymptotic behavior of the form

$$
n_{U, \mathbf{H}}(H) \sim \boldsymbol{\theta}_{\mathbf{H}}(V) H \quad \text { when } H \rightarrow+\infty .
$$


TABLE 2 .

\begin{tabular}{|l|l|l|}
\hline Surface & $S_{5}$ & $S_{6}$ \\
\hline$H$ & 99999 & 99999 \\
\hline$n_{U, \mathbf{H}}(H)$ & 205431 & 115582 \\
\hline$C_{\mathrm{Br}}$ & $1 / 3$ & $1 / 3$ \\
\hline$H^{1}(\mathbf{Q}, \operatorname{Pic}(\bar{V}))$ & 3 & 3 \\
\hline$\zeta_{\mathbf{Q}\left(q^{1 / 3}\right)}^{*}(1)$ & 0.814624 & 1.017615 \\
\hline$\lambda_{3}^{\prime} \boldsymbol{\omega}_{\mathbf{H}}\left(V\left(\mathbf{Q}_{3}\right)\right)$ & 1.333333 & 0.888889 \\
\hline$\lambda_{q}^{\prime} \boldsymbol{\omega}_{\mathbf{H}}\left(V\left(\mathbf{Q}_{q}\right)\right)$ & 0.750000 & 1.000000 \\
\hline$C_{1}$ & 0.954038 & 0.976203 \\
\hline$C_{2}$ & 0.989387 & 0.989279 \\
\hline$C_{3}$ & 0.830682 & 0.306638 \\
\hline $\boldsymbol{\omega}_{\mathbf{H}}(V(\mathbf{R}))$ & 4.921515 & 4.295619 \\
\hline $\boldsymbol{\theta}_{\mathbf{H}}(V)$ & 2.086108 & 1.191539 \\
\hline$n_{U, \mathbf{H}}(H) / \boldsymbol{\theta}_{\mathbf{H}}(V) H$ & 0.984767 & 0.970032 \\
\hline & &
\end{tabular}

\section{ACKNOWLEDGMENTS}

This work was done while both authors were enjoying the hospitality of the Newton Institute for Mathematical Sciences in Cambridge. We have benefitted from conversations with Colliot-Thélène, Heath-Brown, Salberger and SwinnertonDyer. We thank the referee for his useful remarks and suggestions.

The second author was partially supported by NSA Grant MDA904-98-1-0023 and by EPSRC Grant K99015.

\section{REFERENCES}

[BM] V. V. Batyrev et Y. I. Manin, Sur le nombre des points rationnels de hauteur bornée des variétés algébriques, Math. Ann. 286 (1990), 27-43. MR 91g:11069

[BT1] V. V. Batyrev and Y. Tschinkel, Rational points of bounded height on compactifications of anisotropic tori, Internat. Math. Res. Notices 12 (1995), 591-635. MR 97a:14021

[BT2] , Rational points on some Fano cubic bundles, C. R. Acad. Sci. Paris Sér. I Math. 323 (1996), 41-46. MR 97j:14023

[BT3] Tamagawa numbers of polarized algebraic varieties, Nombre et répartition de points de hauteur bornée, Astérisque, vol. 251, SMF, Paris, 1998, pp. 299-340. CMP 99:10

[Be] D. J. Bernstein, Enumerating solutions to $p(a)+q(b)=r(c)+s(d)$, Preprint (1998).

[Co] H. Cohen, A course in computational algebraic number theory, Graduate Texts in Math., vol. 138, Springer-Verlag, Berlin, Heidelberg and New York, 1993. MR 94i:11105

[CT1] J.-L. Colliot-Thélène, Hilbert's theorem 90 for $K_{2}$, with application to the Chow groups of rational surfaces, Invent. Math. 71 (1983), 1-20. MR 85d:14016

[CT2] The Hasse principle in a pencil of algebraic varieties, Number theory (Tiruchirapalli, 1996), Contemp. Math., vol. 210, Amer. Math. Soc., Providence, 1998, pp. 19-39. MR 98g:11075 
[CTKS] J.-L. Colliot-Thélène, D. Kanevsky et J.-J. Sansuc, Arithmétique des surfaces cubiques diagonales, Diophantine approximation and transcendence theory (Bonn, 1985), Lecture Notes in Math., vol. 1290, Springer-Verlag, Berlin, Heidelberg and New York, 1987, pp. 1-108. MR 89g:11051

[CTS] J.-L. Colliot-Thélène et J.-J. Sansuc, La descente sur une variété rationnelle définie sur un corps de nombres, C. R. Acad. Sci. Paris Sér. A-B 284 (1977), 1215-1218. MR 56:5565

[FMT] J. Franke, Y. I. Manin, and Y. Tschinkel, Rational points of bounded height on Fano varieties, Invent. Math. 95 (1989), 421-435. MR 89m:11060

[H-B] D. R. Heath-Brown, The density of zeros of forms for which weak approximation fails, Math. Comp. 59 (1992), 613-623. MR 93a:11055

[IR] K. Ireland and M. Rosen, A classical introduction to modern number theory (second edition), Graduate texts in Math., vol. 84, Springer-Verlag, Berlin, Heidelberg and New York, 1990. MR 92e:11001

[Lac] G. Lachaud, Une présentation adélique de la série singulière et du problème de Waring, Enseign. Math. (2) 28 (1982), 139-169. MR 84b:10072

[Ma1] Y. I. Manin, Le groupe de Brauer-Grothendieck en géométrie diophantienne, Actes Congrès intern. math., Tome 1 (Nice, 1970), Gauthiers-Villars, Paris, 1971, pp. 401-411. MR 55:356

[Ma2] Cubic forms (second edition), North-Holland Math. Library, vol. 4, NorthHolland, Amsterdam, New York and Oxford, 1986. MR 87d:11037

[Pe1] E. Peyre, Hauteurs et mesures de Tamagawa sur les variétés de Fano, Duke Math. J. 79 (1995), no 1, 101-218. MR 96h:11062

[Pe2] - Terme principal de la fonction zêta des hauteurs et torseurs universels, Nombre et répartition de points de hauteur bornée, Astérisque, vol. 251, SMF, Paris, 1998, pp. 259-298. CMP 99:10

[Sa] P. Salberger, Tamagawa measures on universal torsors and points of bounded height on Fano varieties, Nombre et répartition de points de hauteur bornée, Astérisque, vol. 251, SMF, Paris, 1998, pp. 91-258. CMP 99:10

[SaSk] P. Salberger and A. N. Skorobogatov, Weak approximation for surfaces defined by two quadratic forms, Duke Math. J. 63 (1991), no 2, 517-536. MR 93e:11079]

[S-D] P. Swinnerton-Dyer, Counting rational points on cubic surfaces, Classification of algebraic varieties (L'Aquila, 1992) (C. Ciliberto, E. L. Livorni, and A. J. Sommese, eds.), Contemp. Math., vol. 162, AMS, Providence, 1994, pp. 371-379. MR 95d:11078

Institut de Recherche Mathématique Avancée, Université Louis Pasteur et C.N.R.S., 7 rue René-Descartes, 67084 Strasbourg, France

E-mail address: peyre@irma.u-strasbg.fr

Department of Mathematics, University of Illinois in Chicago, 851 South Morgan Street, Chicago IL 60607-7045, USA

E-mail address: yuri@math.uic.edu 